\title{
ARTICLE OPEN \\ Characterization of intrinsic hole transport in single-crystal spiro-OMeTAD
}

\author{
Yuan Li $\mathbb{D}^{1}$, Haoyuan $\mathrm{Li}^{1}$, Cheng Zhong ${ }^{1}$, Gjergji Sini ${ }^{1,2}$ and Jean-Luc Brédas ${ }^{1}$
}

Spiro-OMeTAD remains a prominent hole-transport material in perovskite and solid-state dye-sensitized solar cells. However, an understanding of its intrinsic hole-transport properties is still limited. Here, hole transport in spiro-OMeTAD is systematically characterized on the basis of the recently reported X-ray single-crystal data. An approach combining density functional theory calculations, tight-binding modeling, and kinetic Monte Carlo simulations are exploited to describe the key parameters governing hole transport and to investigate the transport mechanism and hole mobilities in the spiro-OMeTAD single crystal. The results provide insight into: (i) why an anisotropic hole-transport mechanism, with an upper range of intrinsic hole mobilities on the order of $\sim 10^{-3} \mathrm{~cm}^{2} / \mathrm{Ns}$, can be expected in the single crystal; and (ii) how detrimental factors, related to the presence of the spiro motif and of the 4,4'-dimethoxydiphenylamine substituents, limit the intrinsic hole mobilities of the system.

npj Flexible Electronics (2017)1:2; doi:10.1038/s41528-017-0002-0

\section{INTRODUCTION}

As a solid-state hole conductor used in lieu of liquid electrolytes, 2,2',7,7'-tetrakis( $N, N$-di-p-methoxyphenyl-amine) $9,9^{\prime}$-spirobifluorene (spiro-OMeTAD) has long been prominent as the holetransport material (HTM) in solid-state dye-sensitized solar cells. ${ }^{1}$ In these devices, the use of spiro-OMeTAD as the HTM substantially improves the device temporal stability by eliminating the rapid degradation of performance common to redox electrolytes. More recently, spiro-OMeTAD has garnered additional attention because of its widespread application as HTM in the rapidly developing area of perovskite solar cells. ${ }^{2-4}$ In particular, the ionization potential of spiro-OMeTAD matches well with that of the light absorbers (perovskites or dye-sensitizers), and good contact at their interfaces is promoted by the remarkable glassforming properties of spiro-OMeTAD. These advantages make spiro-OMeTAD the most extensively studied organic HTM for hybrid organic-inorganic solar cells with high power conversion efficiency. ${ }^{5-8}$ However, as it is the role of the molecular spiro center to make crystallization difficult, spiro-OMeTAD films are usually amorphous, which results in very low hole mobilities and necessitates doping the materials to improve hole conductivity. This leads to a serious issue limiting the reproducibility and performance of the photovoltaic devices. ${ }^{9}$ Also, the lack of crystalline spiro-OMeTAD, in particular single crystals prepared in a controlled manner, prevents access to the intrinsic holetransport properties of spiro-OMeTAD, the understanding of which is important for improving performance ${ }^{10-12}$ and/or developing alternative organic HTMs. ${ }^{13-15}$

Very recently, significant experimental progress was made in preparing crystalline spiro-OMeTAD. Ganesan et al. reported the first crystal structure of a spiro-OMeTAD complex in which, however, chlorobenzene solvent molecules are also embedded. ${ }^{16}$
While this structure provides some useful information on the molecular packings of spiro-OMeTAD in crystal, the inclusion of the solvent molecules in fact prevents direct contact between the two spiro-OMeTAD molecules within the unit cell. A breakthrough was made by Shi et al., who succeeded in growing pure single crystals of spiro-OMeTAD by an antisolvent crystallization approach. ${ }^{17}$ It was found that the hole mobility of spiroOMeTAD at room temperature is enhanced by three orders of magnitude up to $10^{-3} \mathrm{~cm}^{2} / \mathrm{Vs}$ in the case of the single crystal when compared to spin-coated thin films (all measured in fieldeffect transistor configurations), which demonstrates the importance of mesoscale ordering and crystallinity in improving the performance of spiro-OMeTAD. However, it is important to note that the hole mobilities of the spiro-OMeTAD single crystal $^{17}$ are still much lower than those of prototypical high-mobility organic crystals (e.g., rubrene and pentacene) whose mobilities exceed 1 $\mathrm{cm}^{2} / \mathrm{Vs}$ at room temperature (measured in single-crystal fieldeffect transistor configurations). ${ }^{18,}{ }^{19}$ Given the currently limited understanding of intrinsic hole transport in spiro-OMeTAD, it is important to clarify the underlying mechanism responsible for the hole-mobility enhancement of spiro-OMeTAD in going from thin films to single crystals, as well as the structural bottlenecks detrimental to the intrinsic hole mobilities in the single crystals.

We note that some recent computational studies have been devoted to the description of the hole-transport properties in spiro-OMeTAD. ${ }^{20-23}$ While these investigations provide useful information on hole-mobility predictions in both amorphous and crystalline spiro-OMeTAD, a thorough characterization of the intrinsic hole-transport mechanism in single-crystal spiro-OMeTAD is still missing. In this work, our aim is to provide insight into the intrinsic hole-transport properties of spiro-OMeTAD on the basis of the X-ray single-crystal data. ${ }^{17}$ We employ density functional theory (DFT) calculations in combination with tight-binding (TB)

\footnotetext{
${ }^{1}$ KAUST Solar Center, Physical Science and Engineering Division, King Abdullah University of Science and Technology (KAUST), Thuwal 23955-6900, Saudi Arabia Correspondence: Jean-Luc Brédas (jean-luc.bredas@kaust.edu.sa)

${ }^{2}$ Present address: Laboratoire de Physico-chimie des Polymères et des Interfaces, Université de Cergy-Pontoise, 5 Mail Gay Lussac, Neuville sur Oise, Cergy-Pontoise Cedex 95031, France
}

Received: 6 November 2016 Revised: 21 March 2017 Accepted: 27 March 2017

Published online: 26 September 2017 
(a)
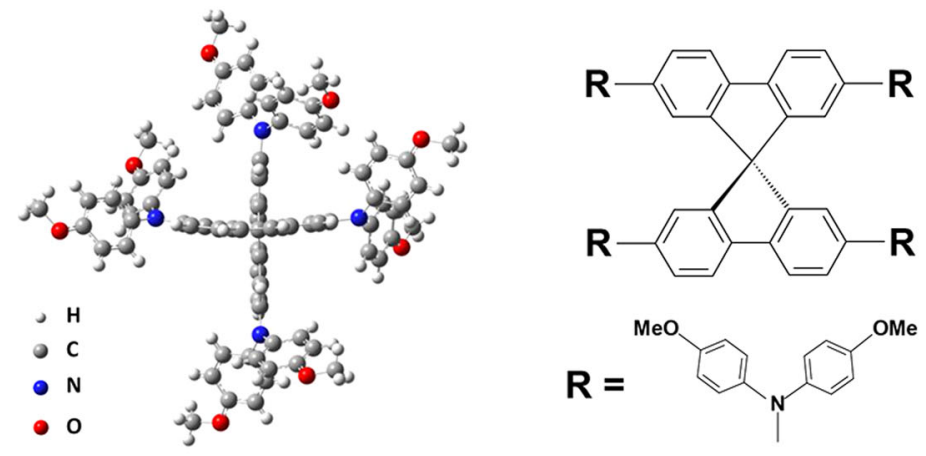

(b)
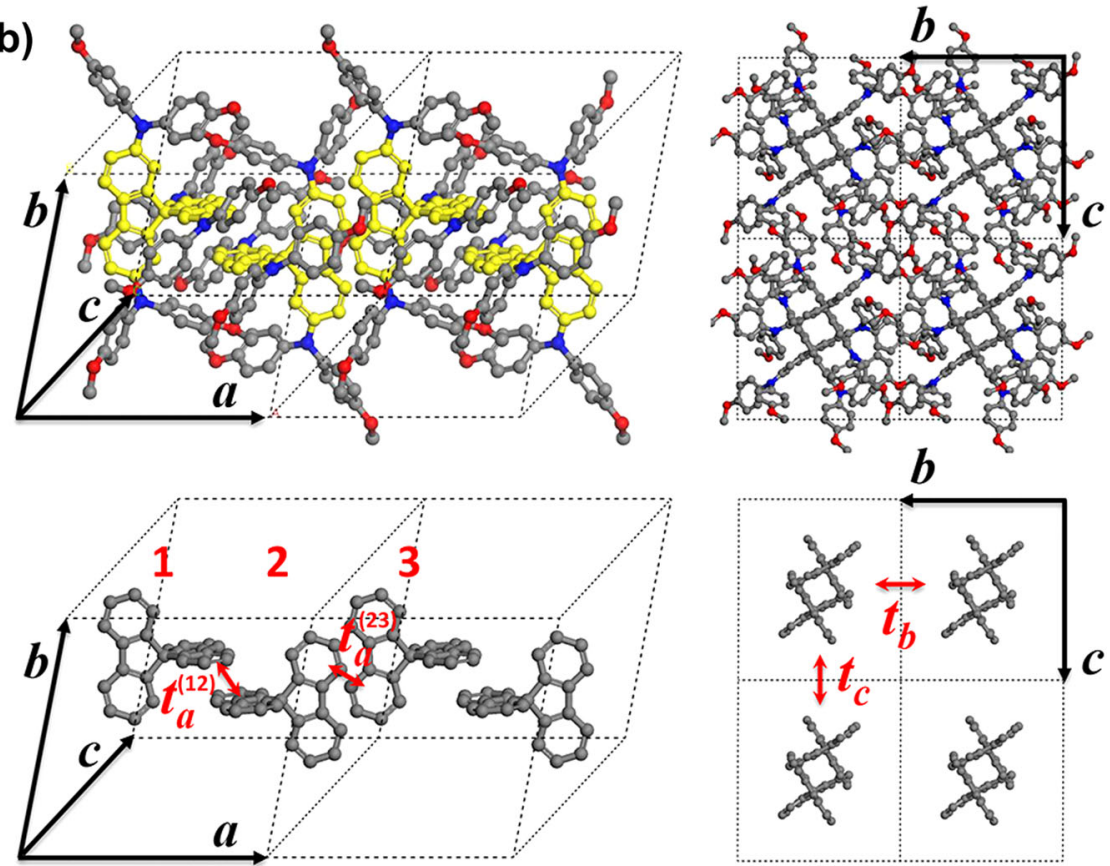

Fig. 1 a Molecular and chemical structures of spiro-OMeTAD; b crystal structure of the spiro-OMeTAD single crystal ${ }^{17}$ (upper panels) and illustration of the stacking of the molecular skeletons, as well as the molecular pairs leading to major transfer integrals (lower panels). In the upper-left panel of $\mathbf{b}$, the bifluorene skeleton of each spiro-OMeTAD molecule is highlighted in yellow

modeling and kinetic Monte Carlo simulations to investigate the hole-transport mechanism and hole mobilities. This approach allows us to characterize the microscopic pathways of hole transport, as well as the inherent bottlenecks limiting the intrinsic hole mobilities of the spiro-OMeTAD single crystal.

\section{RESULTS AND DISCUSSION}

Molecular packing, molecular orbital characteristics, and electronic couplings

Molecular packing. The molecular, chemical, and single-crystal structures of spiro-OMeTAD are illustrated in Fig. 1. As the name implies, the spiro-OMeTAD molecule features a spiro carbon atom linking two orthogonal fluorene-based arms (see Fig. 1a). The spiro-OMeTAD single crystal (see Fig. $1 \mathrm{~b}$ ) belongs to a triclinic space group (details of the crystallographic data were discussed in ref. 17). To gain insight into the correlation between crystal structure and hole-transport properties, we focus on the intermolecular $\pi-\pi$ interactions along different directions of the spiro-OMeTAD single crystal.

Analysis of Fig. 1 shows that the spiro-OMeTAD crystal structure leads to two strikingly different types of intermolecular interactions. The first one corresponds to the $\pi-\pi$ stacking between fluorene planes of adjacent spiro-OMeTAD molecules, which takes place alternatively in two orthogonal orientations (see Fig. 1b, bottom-left panel). This non-continuous $\pi-\pi$ stacking progresses in the $a$ direction, along which efficient hole-transport pathways can be achieved. However, the crystal molecular packings give rise to two structural characteristics that are expected to play a detrimental role for hole transport: (i) The spatial stacking is limited and the equilibrium intermolecular distance is long due to the spiro motif preventing close molecular approaches. The $4,4^{\prime}-$ dimethoxydiphenylamine (OMe-DPA) substituents lead to additional steric hindrance (see Figure $\mathrm{S} 1$ in the Supplementary Information). (ii) The two orthogonal $\pi-\pi$ stacking interactions are not equivalent; indeed, the distance between two spiro carbon atoms is smaller for the intra-unit-cell molecules than that for the inter-unit-cell ones (7.49 and $8.11 \AA$, respectively). An important reason for this difference is that the orientation of the methoxy groups of each spiro-OMeTAD molecule in the crystal is asymmetric with respect to the spiro atom (vide infra) such that equivalent packing for intra-unit-cell and inter-unit-cell molecules cannot be expected. The second type of intermolecular interactions in the spiro-OMeTAD single crystal occurs between the OMeDPA substituents of adjacent molecules along the $b$ and $c$ directions (see Fig. 1b, upper-right panel) with inter-unit-cell distances between spiro carbon atoms of 14.72 and $17.28 \AA$, respectively. However, since these interactions feature poor $\pi-\pi$ 
(a)
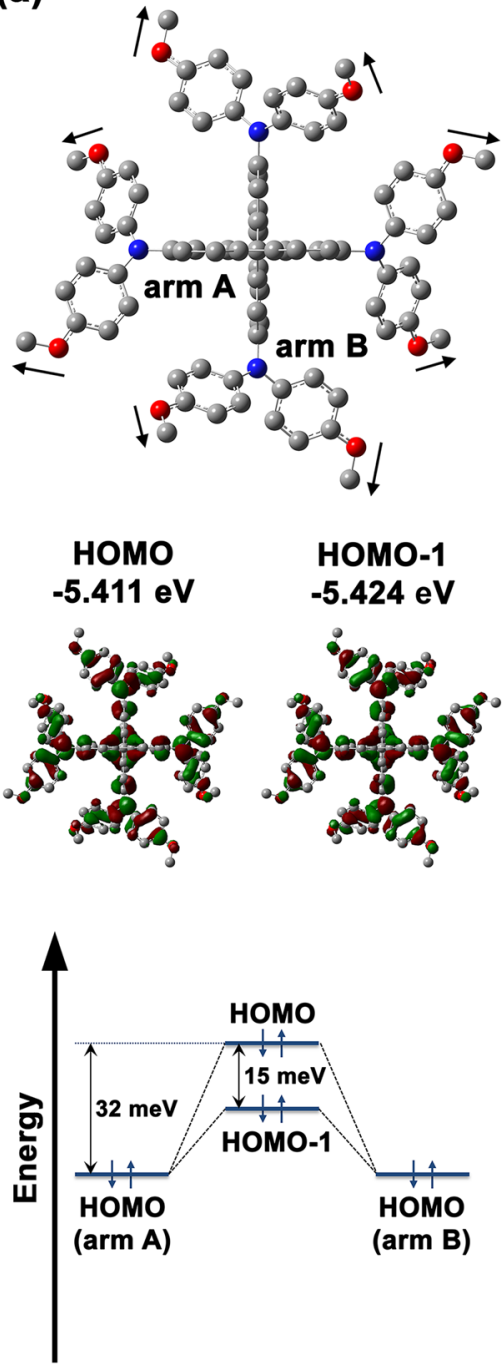

(b)
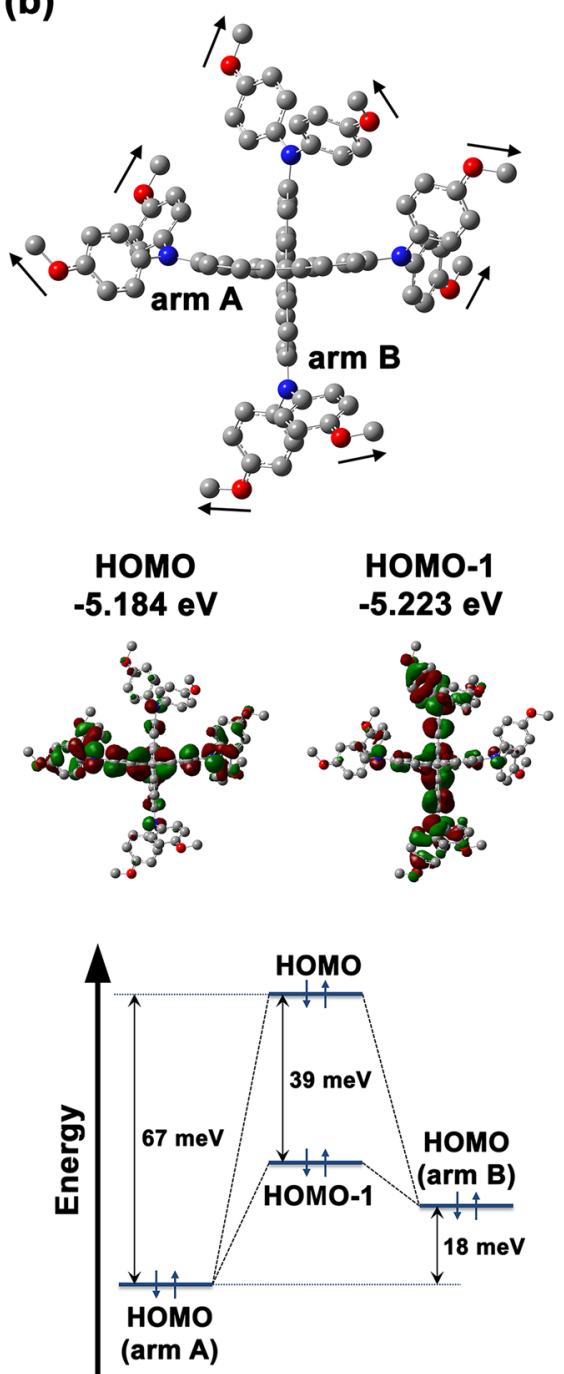

Fig. 2 Comparison of the molecular geometry, DFT (Opt- $\omega$ B97X-D/6-31 G**)-estimated wave functions and energy levels of HOMO and HOMO-1, and DFT ( $\omega$ B97X/TZ2P)-estimated interaction between the fragment HOMOs of the two arms for: a an ideal spiro-OMeTAD molecule in gas phase; and $\mathbf{b}$ a molecule extracted from the spiro-OMeTAD crystal. The arrows in the top panels denote the orientation of the methoxy groups. All the molecules are in their neutral ground state

stackings as a result of the propeller-shape geometry of the DPA moieties around the nitrogen atoms, they are not expected to have significant contributions to the hole-transport properties of the spiro-OMeTAD single crystal (vide infra).

Molecular orbital characteristics. Before discussing the electronic couplings between the molecular orbitals involved in hole transport in the spiro-OMeTAD crystal, it is useful to describe the nature of the highest-occupied molecular orbital (HOMO) and HOMO-1 and the origin of their characteristics. To this end, it is very instructive to start with an "ideal" (that is, geometrically optimized for the ground state in the gas phase), isolated spiroOMeTAD molecule where the two arms (defined as arm A and arm B) are perfectly symmetric with respect to the spiro atom, as shown in the top panel of Fig. 2a. The presence of two symmetric arms gives rise to nearly degenerate HOMO and HOMO-1 levels with an energy difference of about $13 \mathrm{meV}$. Fragment calculations (in which the molecular HOMO and HOMO-1 are built up from the fragment HOMOs of arm A and arm B, see the bottom panel in Fig. 2a) demonstrate that the HOMOs of the two arms are energetically identical (because of their identical geometry); their electronic couplings through the spiro atom lead to equal contributions to the HOMO and HOMO-1 of the whole molecule. In this instance, the wave functions of the molecular HOMO and HOMO- 1 are fully delocalized over both arms (see the middle panel in Fig. 2a). It is interesting to note that the HOMOs of the two (isolated) arms are lower in energy than both HOMO and HOMO- 1 of the whole molecule. In fact, each arm has an electric dipole moment of about 1.7 Debye, while the whole molecule has none as a result of its symmetry (see Table S1 in the Supplementary Information). The interactions among the arm dipole moments when the two arms are connected via the spiro atom induce intramolecular electronic polarization effects that destabilize the molecular HOMO and HOMO-1 levels.

We now turn to the case of an actual spiro-OMeTAD molecule extracted from the single crystal. Because of the influence of the packing forces, the geometry of a spiro-OMeTAD molecule in the crystal exhibits important differences between the two arms due to the different intermolecular packings/distances along the $a$ direction of the crystal. As a result, the orientations of the methoxy groups vary with respect to the spiro atom and the diphenylamine moieties and bifluorene skeletons distort in a non-symmetric fashion, as illustrated in the top panel of Fig. $2 \mathrm{~b}$. Indeed, results of fragment calculations show that the HOMO of arm A is about 18 
Table 1. DFT estimates of the transfer integrals $t_{a}^{(i j)}$ (in meV, with $i, j=1,2$, or 3 ) for molecular pairs extracted from the spiro-OMeTAD crystal illustrated in Fig. $1 \mathrm{~b}$ for the couplings between the HOMOs $(\mathrm{H})$, the $\mathrm{HOMO}-1 \mathrm{~s}(\mathrm{H}-1)$, and the $\mathrm{HOMO}$ and $\mathrm{HOMO}-1$ (H/H-1) of the individual molecules. The calculations were performed at the Opt- $\omega$ B97X-D/6-31 $\mathrm{G}^{* *}$ and (in parentheses) B3LYP/6-31 G** levels of theory

\begin{tabular}{|c|c|c|c|c|}
\hline & $t_{a}^{(12)}$ & $t_{a}^{(23)}$ & $t_{b}$ & $t_{c}$ \\
\hline $\mathrm{H}$ & $9.3(8.6)$ & $37.6(36.2)$ & $-1.6(-1.5)$ & $-0.3(-0.3)$ \\
\hline $\mathrm{H}-1$ & $33.4(38.4)$ & $7.0(5.2)$ & $0.5(0.5)$ & $-0.8(-0.8)$ \\
\hline $\mathrm{H} / \mathrm{H}-1$ & $-15.2(-16.2)$ & $17.0(14.6)$ & $0.2(0.2)$ & $0.5(0.5)$ \\
\hline
\end{tabular}

meV lower in energy than that of arm B as a result of the geometry deformations (see the bottom panel in Fig. 2b). The geometry differences between the two arms cause the HOMO and HOMO-1 wave functions of the spiro-OMeTAD molecule to localize on one of the two arms (see the middle panel in Fig. 2b). Moreover, the electric dipole moments of arm A and arm B are estimated to be about 4.5 Debye and 3.0 Debye, respectively, leading to more prominent intramolecular polarization effects as compared with the case of the ideal isolated spiro-OMeTAD molecule. These large intramolecular polarization effects result in an energy difference of about $39 \mathrm{meV}$ between the HOMO and HOMO-1 levels of the spiro-OMeTAD molecule. It is also important to note that the ionization potential of the spiro-OMeTAD molecule taken from the crystal is about $0.23 \mathrm{eV}$ smaller than that of the ideal isolated molecule (see the middle panel in Fig. $2 a$ and $b$ ). This result points to the importance of the molecular geometry in determining the level alignment at organic/inorganic interfaces of hybrid solar cells where the geometry of spiro-OMeTAD molecules can be easily affected. $^{5}$

Electronic couplings. We now proceed to estimate the electronic couplings (transfer integrals) for representative molecular pairs along all three axes of the spiro-OMeTAD crystal (see Fig. 1b, lower panels). Here, both HOMO and HOMO-1 have to be taken into account in these calculations as a result of their quasi-degeneracy discussed above. The DFT-calculated electronic couplings are collected in Table 1. Substantial transfer integrals are obtained along the $a$ axis, with the largest value of ca. $40 \mathrm{meV}$ due to the $\pi-$ $\pi$ stacking between the fluorene planes of adjacent molecules. However, the transfer integrals along the remaining two axes, where adjacent molecules are coupled through only part of the OMe-DPA substituents (see Fig. 1b), are vanishingly small. This underlines that strongly anisotropic (quasi-one-dimensional) holetransport properties can be expected in the spiro-OMeTAD single crystal. Because the HOMO and HOMO-1 wave functions are mainly localized on one of the two arms, the associated transfer integrals for the molecular pair within the unit cell are calculated to be much different from those between neighboring unit cells. There occurs alternations in transfer integrals along the $a$ direction of the crystal, and the characteristics of these alternations are different for the HOMO and HOMO-1 levels. For instance, the transfer integral associated with HOMO [HOMO-1] is small [large] for the intra-unit-cell pair and the reverse holds true for the interunit-cell pair. Interestingly, in addition to the electronic couplings associated solely with HOMO or HOMO-1, there exist substantial cross electronic couplings (denoted $\mathrm{H} / \mathrm{H}-1$ in Table 1) between HOMO and HOMO-1. These couplings, as will be shown, play a critical role in the formation of hole-transport pathways in the spiro-OMeTAD crystal.

It is also instructive to briefly discuss the transfer integrals and their potential effects on hole transport in the case of the cocrystal of spiro-OMeTAD with chlorobenzene. ${ }^{16}$ We note that, while also belonging to a triclinic space group, the structure of the co-crystal is distinctly different from that of the pure spiroOMeTAD crystal. For instance, due to the presence of the chlorobenzene molecules, the center-to-center distance between the two spiro-OMeTAD molecules within the unit cell of the cocrystal is $13.64 \AA$, compared to $7.49 \AA$ in the case of the pure spiroOMeTAD crystal (see Figure S3 in the Supplementary Information). In this context, the transfer integrals as a result of direct couplings between the spiro-OMeTAD molecules within the unit cell are calculated to be vanishingly small; thus, the presence of the chlorobenzene molecules has definitely to be taken into account when accounting for the hole-transport properties of the spiroOMeTAD-chlorobenzene co-crystal. The transfer integrals for the inter-unit-cell spiro-OMeTAD molecules along all three axes are also significantly different when comparing the co-crystal with the pure crystal (see Table S2 in the Supplementary Information). These results demonstrate that the inclusion of solvent molecules in the case of spiro-OMeTAD-chlorobenzene co-crystal very strongly impacts the molecular packings and electronic couplings; as a result, the intrinsic hole transport of spiro-OMeTAD cannot be achieved in the co-crystal.

\section{Electronic band structures}

In order to analyze the hole-transport mechanism, we estimated the electronic band structures of the spiro-OMeTAD crystal by means of both DFT calculations and tight-binding modeling. The electronic band dispersions along $\Gamma \rightarrow X$, which to a very good approximation corresponds to the $a$ direction in real space, are illustrated in Fig. 3a. The band structures along other pathways in reciprocal space and the total density of electronic states (which were partly reported in ref. 17) can be found in the Supplementary Information. Figure 3a illustrates that, while the width of the overall top valence band spans more than $200 \mathrm{meV}$, it splits into four sub-bands separated by sizable energy gaps, which is due to the presence of four fluorene-based arms within a unit cell. The energy separation between the topmost sub-band and the next sub-band is about $85 \mathrm{meV}$, which is much larger than thermal energy at room temperature (ca. $26 \mathrm{meV}$ ). Thus, the hole carriers are expected to mostly thermally populate the topmost sub-band near room temperature. Since the topmost sub-band is almost dispersionless along all directions (see Figure S4 in the Supplementary Information), band-like transport cannot be expected in the spiro-OMeTAD crystal.

We also evaluated the intramolecular reorganization energy $\lambda_{\text {reorg }}$ of spiro-OMeTAD. The value calculated at the DFT Opt$\omega B 97 X-D / 6-31 G^{* *}$ level of theory is about $310 \mathrm{meV}$. We note that this value of $\lambda_{\text {reorg }}$ is much larger than those reported in previous studies based on the B3LYP functional (e.g., $131 \mathrm{meV}$ in ref. 21 and $160 \mathrm{meV}$ in ref. 22). This large difference is caused by the delocalization error (i.e., spurious delocalization of electron densities and overestimation of conjugation) inherent to the B3LYP functional. ${ }^{24}$ This issue can be overcome by using longrange corrected functionals with tuned range-separation parameters, such as the one employed in the calculations here. ${ }^{24}$ Since the value of $\lambda_{\text {reorg }}$ is much larger than that of any transfer integral, it is expected that hole transport in the spiro-OMeTAD crystal is governed by hopping of (small) polarons. ${ }^{25}$

To gain a better understanding of the intrinsic hole-transport properties, we developed a one-dimensional tight-binding model ${ }^{26}$ 
(b)

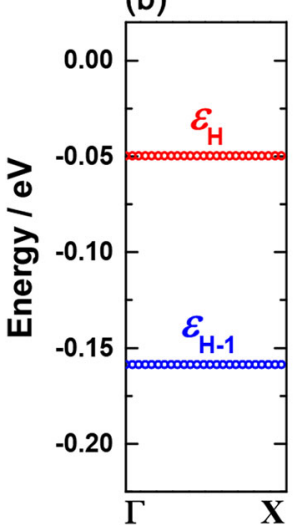

(c)

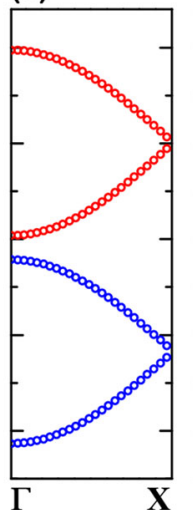

(d)

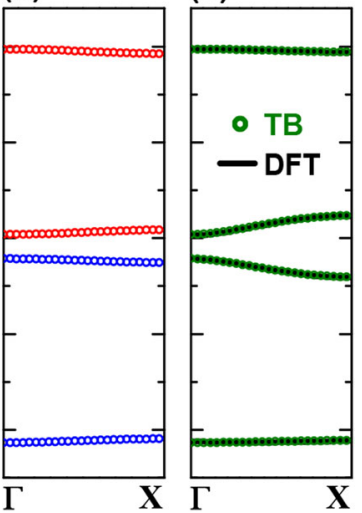

Fig. 3 Tight-binding (TB) band structure along $\Gamma \rightarrow X$ of the spiroOMeTAD crystal with different values of the model parameters (see TB Values in Table S3): a full parameters; $\mathbf{b}$ site energies only; c site energies and average values of transfer integrals associated with HOMO and HOMO-1; d full parameters except for zero cross electronic couplings. The DFT band structure is also displayed in a The crystallographic coordinates of $\Gamma$ and $X$ are $(0,0,0)$ and $(0.5,0$, $0)$, respectively; the Fermi level is taken as reference (set at zero)

(detailed in the Supplementary Information) to quantitatively estimate the electronic properties of the spiro-OMeTAD crystal. All the model parameters [that is, the site energies $\varepsilon$ for the two molecules in the unit cell and the transfer integrals $t^{(i j)}$ (with $i, j=1$, 2 , or 3, see the lower-left panel in Fig. 1b) for both HOMO and HOMO-1, intra- and inter-unit-cell] were evaluated by fitting the TB band structures to those of the DFT calculations along a specific direction in reciprocal space. The values of the model parameters obtained by fitting the band structure along $\Gamma \rightarrow X$, as illustrated in Fig. 3a, can be found in the Supplementary Information (Table S3).

To understand the origin of the characteristics of the band structures, we calculated the band dispersions along $\Gamma \rightarrow X$ by artificially modifying the values of the model parameters (TB Values in Table S3) within our tight-binding model. In particular, we considered three cases in which increasing numbers of transfer integrals are progressively switched on: (i) all the transfer integrals are set to zero (i.e., only the site energies are considered); (ii) the transfer integrals associated with $\mathrm{HOMO}$ and HOMO-1 are introduced and given values corresponding to the average of the values of the transfer integrals for intra-unit-cell and inter-unitcell $\tilde{t}_{\mathrm{H}[\mathrm{H}-1]}=\left(t_{\mathrm{H}[\mathrm{H}-1]}^{(2)}+t_{\mathrm{H}[\mathrm{H}-1]}^{(23)}\right) / 2$ (i.e., no transfer integral alternation is considered along the $a$ direction); (iii) all parameters are used except for the cross electronic couplings that are neglected $\left(t_{\mathrm{H} / \mathrm{H}-1}=0\right)$. The band structures corresponding to these three specific cases are displayed in Fig. 3b-d, respectively. As expected, Fig. $3 \mathrm{~b}$ shows no band dispersion due to the absence of electronic couplings. The two flat bands just correspond to the HOMO and HOMO-1 levels of the individual molecules in the crystal. When the average values (no alternation) of the transfer integrals $\left(\tilde{t}_{\mathrm{H}[\mathrm{H}-1]}=24 \mathrm{meV}\right)$ are introduced, see Fig. 3c, a considerable dispersion of about $100 \mathrm{meV}$ is obtained for both the HOMO and HOMO-1 bands. However, when the alternations in the transfer integrals are introduced, as shown in Fig. 3d, the HOMO and HOMO-1 bands both split into two sub-bands with small dispersions. Further inclusion of the cross electronic couplings (see Fig. 3a) does not significantly change the band structures. These results allow us to understand that the formation of nearly dispersionless bands, which is clearly detrimental to hole transport, is mainly caused by the alternations in the magnitudes of the transfer integrals along the $a$ direction of the spiro-OMeTAD crystal.
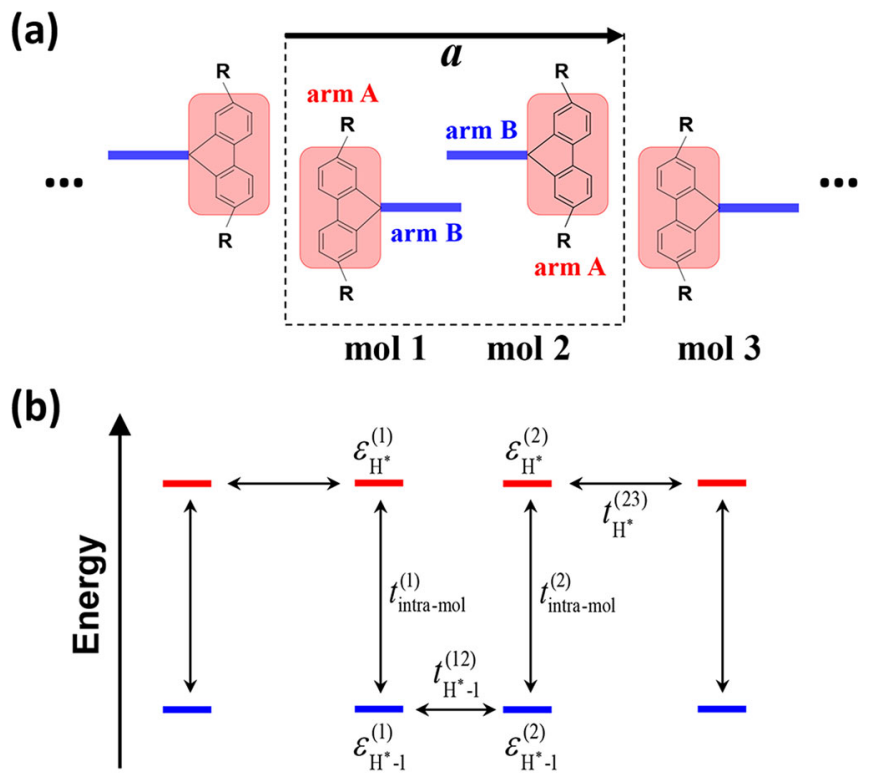

Fig. 4 Schematic diagrams of $\mathbf{a}$ the molecular packing and $\mathbf{b}$ the effective pathway for hole transport along the $a$ direction of the spiro-OMeTAD crystal. In a, the dashed rectangle denotes the unit cell and the solid arrow represents the lattice constant $(a=13.7 \AA)$

It is interesting to note that the values of the model parameters (both transfer integrals and site energies) obtained in the tightbinding model markedly differ from the DFT results on isolated molecules. For instance, the $\mathrm{HOMO} / \mathrm{HOMO}-1$ energy difference in the TB modeling is about $109 \mathrm{meV}$, which is much larger than the DFT result of about $39 \mathrm{meV}$ for an isolated molecule. The major reason for the differences is that electrostatic interactions between spiro-OMeTAD molecules in the crystal are effectively included in the TB modeling (via the DFT estimation of band structures on which the TB fitting was based). This result again underlines the importance of intermolecular polarization effects in the solid state, ${ }^{27,} 28$ in impacting the electronic properties of spiroOMeTAD. These intermolecular polarization effects are related to at least two factors. On the one hand, a permanent dipole moment as large as 5.8 Debye is evaluated for each spiro-OMeTAD molecule in the crystal (see Table S1 in the Supplementary Information). On the other hand, the molecular spiro motifs lead to different crystal environments impacting the HOMO and HOMO-1 wave functions localized on different arms of each molecule.

Hole-transport pathways, properties, and bottlenecks

Hole-transport pathways and properties. In order to visualize the hole-transport pathways, we consider a stack of molecules along the $a$ direction of the spiro-OMeTAD crystal, as schematically illustrated in Fig. 4a. Due to the presence of the cross electronic couplings $t_{\mathrm{H} / \mathrm{H}-1}$, hole-transport pathways involving both the HOMO and HOMO-1 channels can be expected in the crystal. In order to obtain a simple (but reliable) picture, it was found useful to apply a transformation procedure, leading to a new set of molecular orbitals referred to as $\mathrm{HOMO}^{*} \mathrm{~s}$ and $\mathrm{HOMO}^{*}-1 \mathrm{~s}$, in which the cross electronic couplings are eliminated (i.e., $t_{\mathrm{H} / \mathrm{H}-1}=0$, see the Supplementary Information); in this way, an effective holetransport pathway corresponding to a single channel is obtained, as illustrated in Fig. 4b (the effective values of the model parameters after the transformation are collected in Table S3). Since the transformation effectively incorporates the impact of $t_{\mathrm{H} /}$ $\mathrm{H}-1$ into other parameters, the values of the new parameters associated with $\mathrm{HOMO}^{*} \mathrm{~s}$ and $\mathrm{HOMO}^{*}-1 \mathrm{~s}$ are all changed with 


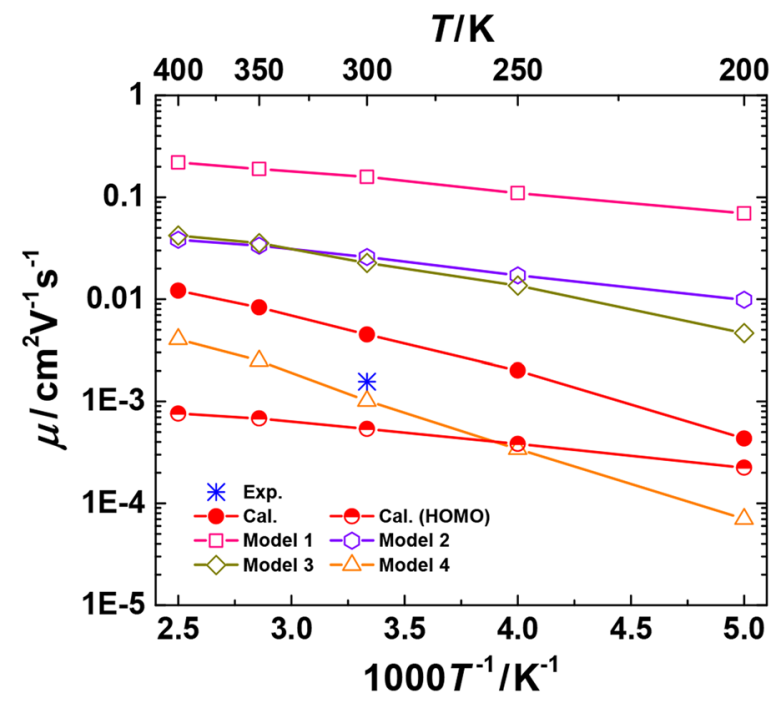

Fig. 5 Kinetic Monte Carlo simulations of the temperaturedependent hole mobilities. These are calculated for: (i) the spiroOMeTAD crystal by using the full set of parameters (Cal.) and by considering only the HOMO channel [Cal. (HOMO)], as well as (ii) four model systems in which homogeneous transfer integrals (Model 1), the absence of intermolecular electronic polarization effect (Model 2), smaller intramolecular reorganization energy (Model 3), and a model crystal with $R=$ diphenylamine (Model 4 ) were considered, respectively. For comparison, the experimental hole mobility at room temperature (Exp.) ${ }^{17}$ is also displayed

respect to those of the original ones; for instance, the values of $t_{\mathrm{H}^{*}-1}^{(12)}$ and $t_{\mathrm{H}^{*}}^{(23)}$ are increased with respect to their counterparts, $t_{\mathrm{H}^{*}-1}$ and $t_{\mathrm{H}^{*}}$
while $t_{\mathrm{H}^{*}}^{(12)}$ and $t_{\mathrm{H}^{*}-1}^{(23)}$ are both reduced to zero, which completely blocks the transport channels of both $\mathrm{HOMO}^{*}$ and $\mathrm{HOMO}^{*}-1$ (see Fig. 4b). In this context, $t_{\mathrm{H} / \mathrm{H}-1}$ is seen to play a detrimental role to hole transport (which cannot have been concluded based on the original parameters). However, interestingly, the transformation introduces a new parameter $t_{\text {intra-mol }}$ describing the intramolecular coupling between the $\mathrm{HOMO}^{*}$ and $\mathrm{HOMO}^{*}-1$ of each molecule; the appearance of $t_{\text {intra-mol }}$ is the result of the non-orthogonality between the $\mathrm{HOMO}^{*}$ and $\mathrm{HOMO}^{*}-1$ of each molecule (see the Supplementary Information). Importantly, $t_{\text {intra-mol }}$ represents the key parameter governing the intra-molecular charge transfer between the two arms of the individual molecules, which is critical to reconnect the HOMO ${ }^{*}$ and $\mathrm{HOMO}^{*}-1$ (see Fig. 4b). Thus, $t_{\mathrm{H} / \mathrm{H}-1}$, via the role played by $t_{\text {intra-mol, }}$ here provides a positive contribution to the hole-transport properties of the system.

In order to quantitatively estimate the hole-transport properties of the spiro-OMeTAD crystal, we evaluated the hole mobilities by means of kinetic Monte Carlo simulations (detailed in the Supplementary Information). ${ }^{29,} 30$ The effective hole-transport pathway along the $a$ direction of the crystal (see Fig. 4b) was considered, and the values of the model parameters used in the calculations can be found in the Supplementary Information (Table S4). The calculated hole mobilities, $\mu$, as a function of temperature, $T$, are illustrated in Fig. 5. As expected, the hole mobilities display a thermally activated behavior; they approximately follow a $\ln \mu \propto$ $-T^{-1}$ relationship (more accurate analytical results of the mobilitytemperature relationship were reported in Reference 31), consistent with the classical Marcus equation ${ }^{32}$ used in the hopping rate calculations (see Eq. 1). The hole mobility at $300 \mathrm{~K}$ is estimated to be $4.5 \times 10^{-3} \mathrm{~cm}^{2} / \mathrm{Vs}$, which is in very good agreement with the experimental result of about $1.3 \times 10^{-3} \mathrm{~cm}^{2} / \mathrm{Ns}$ (measured for a single-crystal spiro-OMeTAD field-effect transistor). ${ }^{17}$ Given that extrinsic factors (e.g., impurities and/or disorder effects) are difficult to prevent experimentally and that all key parameters governing intrinsic hole transport have been accounted for in our calculations, it is reasonable to conclude that the upper range of the intrinsic hole mobilities in the spiro-OMeTAD single crystal is on the order of a few $10^{-3} \mathrm{~cm}^{2} / \mathrm{Vs}$.

We also calculated the hole mobilities of spiro-OMeTAD by considering only the HOMO transport channel based on the original model parameters (see Table S4). The results are also displayed in Fig. 5. The hole mobilities near room temperature are reduced by about one order of magnitude with respect to those of the full-parameter calculations and are smaller than the experimental value. This demonstrates that the HOMOs and HOMO-1s of the individual molecules, rather than just the HOMOs, have to be included in the description of hole transport in the spiro-OMeTAD crystal.

Hole-transport bottlenecks. Since the hole mobilities calculated for the spiro-OMeTAD crystal are some 3-4 orders of magnitude lower than those of prototypical high-mobility organic crystals such as rubrene and pentacene, ${ }^{18,19}$ it is important to analyze the major bottlenecks associated with the molecular and crystal structures that are detrimental to the hole-transport properties of spiro-OMeTAD. As discussed in Section Molecular packing, the molecular packings, which directly impact the values of the key parameters governing hole transport, are determined by both the spiro motif and the nature of the $4,4^{\prime}$-dimethoxydiphenylamine substituents of the individual molecules.

Characteristic features of spiro-OMeTAD lead to three important factors that negatively impact hole transport.

(1) Large alternations in transfer integrals: These alternations are caused by the localization of the HOMO and HOMO-1 wave functions of the spiro-OMeTAD molecules that are geometrically distorted in the crystal (see Section Electronic couplings). As the main origin for the dispersionless band structure, such large alternations in transfer integrals are expected to greatly limit the hole mobilities of the spiro-OMeTAD crystal (see below).

(2) Large electronic polarization effects: The polarization in the spiro-OMeTAD crystal has both intermolecular and intramolecular contributions, coming from electrostatic interactions between the electric dipole moments of the individual molecules and their arms, respectively. The presence of large polarization effects is detrimental to hole transport for two reasons: (i) Intermolecular polarization in the crystal significantly increases the HOMO/ HOMO-1 energy difference of the individual molecules (see Section Electronic band structures), thereby reducing the contributions of the HOMO-1 channels to the hole transport. (ii) Intramolecular polarization leads to localization of the HOMO and HOMO-1 wave functions of the spiro-OMeTAD molecules on separate arms (see Section Molecular orbital characteristics).

(3) Large intramolecular reorganization energy: The $\lambda_{\text {reorg }}$ value calculated for spiro-OMeTAD $(310 \mathrm{meV})$ is about 2 to 3 times as large as that of rubrene $(159 \mathrm{meV})^{33}$ and pentacene $(97 \mathrm{meV})^{34}$. Such a large $\lambda_{\text {reorg }}$ value considerately decreases the hopping rate of polarons and is thus detrimental to the hole-transport properties of the spiro-OMeTAD crystal.

In the context of our present discussion, it is useful to evaluate the mobilities of four model systems obtained by changing the values of (some of) the relevant parameters of the spiro-OMeTAD crystal. In Model 1, the alternations in transfer integrals along the $a$ direction of the crystal are removed by replacing the transfer integrals associated with $\mathrm{HOMO}$ and $\mathrm{HOMO}-1$ with their average values (corresponding to the system displayed in Fig. 3c). In Model 2 , the intermolecular polarization is removed by using the values of the transfer integrals and site energies obtained from isolated molecules instead of the crystal. In Model 3, the value of $\lambda_{\text {reorg }}$ is reduced by about $50 \%$ (to be as small as that of rubrene). In Model 4 , the substituents of the spiro-OMeTAD molecules in the crystal are modified by removing all the methoxy groups while keeping the same crystal structure as in the spiro-OMeTAD single crystal. (It should be borne in mind that the model crystals are based on the 
same crystal parameters as those of the spiro-OMeTAD crystal. Thus, all the results obtained for the model crystals are useful only in comparison to the experimental spiro-OMeTAD crystal.) The values of all the parameters used in these calculations can be found in the Supplementary Information (Table S4), and the mobility results for these four model systems are illustrated in Fig. 5.

As seen from Fig. 5, the mobilities calculated in the cases of Models 1, 2, and 3 are all greatly improved when comparing to the case of the real spiro-OMeTAD crystal. In particular, the mobility at $300 \mathrm{~K}$ in the case of Model 1 is estimated to be $0.16 \mathrm{~cm}^{2} / \mathrm{Ns}$, which is two orders of magnitude larger than that measured and calculated for the actual spiro-OMeTAD crystal. This result indicates that, at least for the three factors discussed above, the presence of large transfer integral alternations in the crystal represents the most critical factor detrimental to the holetransport properties of spiro-OMeTAD. In addition, the mobility also considerably increases in the cases of Models 2 and $3(2.6 \times$ $10^{-2}$ and $2.3 \times 10^{-2} \mathrm{~cm}^{2} \mathrm{Ns}$ at $300 \mathrm{~K}$, respectively), which points to the role of large intermolecular polarization effects and large $\lambda_{\text {reorg }}$ in limiting the hole mobilities of spiro-OMeTAD. Interestingly, in contrast to the three cases mentioned above, the mobilities in the case of Model 4 become even smaller than those of the actual spiro-OMeTAD system. The removal of the methoxy groups in the case of Model 4 is found to have opposite effects. On the one hand, it leads to delocalized $\mathrm{HOMO}$ and $\mathrm{HOMO}-1$ wave functions as well as smaller electric dipole moments. On the other hand, it gives rise to a larger $\lambda_{\text {reorg }}$ (about $454 \mathrm{meV}$ ). (The reorganization energy in the case of $R=$ diphenylamine is larger because, in the absence of the methoxy group, the transformation from an aromatic (small bond-length alternation) to a quinoidal (large bond-length alternation) geometry of the phenyl moiety upon oxidation is more prominent; thus, a larger geometry relaxation is induced.) The combination of these effects lead to an overall decrease of the mobilities in the case of Model 4. It is useful to keep in mind that the results of Model 4 were obtained by assuming that the crystal structure remains unchanged after the removal of the methoxy groups, which might not hold true in reality since the crystal structure itself would then relax. In any event, the results of Model 4 underline that the three factors detrimental to hole mobility in the spiro-OMeTAD crystal are not independent but are correlated (in different ways) in determining the properties of the system. Moreover, because of the different intermolecular distances within and between unit-cells, alternations in transfer integrals and dispersionless band structures are also present in the case of Model 4, in spite of the delocalization of the HOMO and HOMO- 1 wave functions (see Table S4 and Figure S4 in the Supplementary Information). This points to the importance of optimizing both molecular and crystal structures of spiro-OMeTAD types of systems in order to improve the holetransport properties.

\section{CONCLUSIONS}

We have investigated the intrinsic hole-transport properties of spiro-OMeTAD, a benchmark hole-transport material intensively studied in perovskite and solid-state dye-sensitized solar cells. Based on the experimental single crystal data obtained very recently, ${ }^{17}$ we have determined the key parameters (transfer integrals, site energies, and reorganization energies) governing intrinsic hole transport and the hole mobilities by means of density functional theory calculations, tight-binding modeling, and kinetic Monte Carlo simulations.

The major conclusions we can draw are as follows:

(i) The $\pi-\pi$ stacking of the fluorene planes of adjacent spiroOMeTAD molecules gives rise to substantial transfer integrals only along the $a$ axis, leading to strong anisotropic (quasi-one-dimensional) hole-transport properties in the spiro-OMeTAD crystal. (ii) Because of the spiro motif and the non-symmetric geometry distortions of the two arms in the spiro-OMeTAD molecules, the $\pi-\pi$ stacking characteristics alternate along the $a$ direction, leading to a dispersionless electronic (top) valence band in the crystal.

(iii) The upper range for the intrinsic hole mobilities near room temperature in the spiro-OMeTAD crystal is predicted to be on the order of $\sim 10^{-3} \mathrm{~cm}^{2} / \mathrm{Ns}$, which is 3-4 orders of magnitude lower than the hole mobilities of prototypical high-mobility organic crystals such as rubrene and pentacene.

(iv) The combined effects of the spiro motif and the 4,4'dimethoxydiphenylamine substituents lead to three important factors detrimental to hole transport, i.e., large alternations in the magnitude of the transfer integrals along the $a$ direction of the crystal, large electronic polarization effects, and large intramolecular reorganization energy. Overcoming these detrimental factors could increase the hole mobilities of spiro-OMeTAD by about $1-2$ orders of magnitude.

This work has allowed us to uncover the features of the molecular and crystal structures that most impact the intrinsic hole-transport properties of spiro-OMeTAD and can help guide the design of the spiro-OMeTAD-like materials with improved hole-transport characteristics.

\section{METHODS}

\section{Density functional theory calculations}

The molecular electronic properties (molecular orbitals, transfer integrals, reorganization energies, and electric dipole moments) were calculated with the Gaussian09 package ${ }^{35}$ by using the non-empirically tuned longrange-corrected $\omega \mathrm{B} 97 \mathrm{X}-\mathrm{D}$ functional, ${ }^{36}$ where the optimal value of the range-separation parameter $\omega$ is obtained by minimizing the energy difference between the HOMO and ionization potential (that is, the difference in total energy between the cationic and neutral ground states of the molecule for a fixed geometry) of a spiro-OMeTAD single molecule. It is important to note that the use of the tuned long-range-corrected functional largely overcomes the delocalization error inherent to semilocal and global hybrid functionals, in particular in the case of spiro structures. ${ }^{24}$ More details on the optimization of the range-separation parameter have been well documented in the literature. ${ }^{37}$ The electronic band structures were calculated with the CRYSTAL14 package ${ }^{38}$ by using the B3LYP functional (we note that long-range-corrected functionals with tunable $\omega$ are currently not available for periodic systems and those with default values of $\omega$ lead to convergence issues with the spiro-OMeTAD single crystal used in our calculations). However, given that the transfer integrals calculated at the B3LYP level agree very well with the Opt- $\omega$ B97X-D results (see Table 1), the band structures calculated with the B3LYP functional are reliable. The $6-31 \mathrm{G}^{* *}$ basis set was used in all calculations. The fragment calculations performed to obtain the results illustrated in the lower panels of Fig. 2 were carried out with ADF Modeling Suite ${ }^{39-41}$ at the DFT $\omega B 97 X /$ TZ2P level of theory.

\section{Mobility calculations}

Within the small-polaron hopping approximation, the hole mobilities were estimated via kinetic Monte Carlo simulations (that have been widely exploited to depict such a hopping transport regime). ${ }^{29}$ The hopping rates were calculated with the semi-classical Marcus equation: ${ }^{25,} 32$

$\kappa_{i j}=\frac{t^{2}}{\hbar}\left(\frac{\pi}{\lambda_{\text {reorg }} k_{\mathrm{B}} T}\right)^{1 / 2} \exp \left[-\frac{\left(\lambda_{\text {reorg }}+\varepsilon_{j}-\varepsilon_{i}\right)^{2}}{4 \lambda_{\text {reorg }} k_{\mathrm{B}} T}\right]$,

where $\lambda_{\text {reorg }}$ denotes the reorganization energy; $t$, the transfer integral; $\varepsilon$, the site energy (i.e., the energy of $\mathrm{HOMO}^{*}$ or $\mathrm{HOMO}^{*}-1$ ); $T$, the temperature; $k_{\mathrm{B}}$, the Boltzmann constant; and $\hbar$, the Planck constant. All the key parameters $\left(t, \lambda_{\text {reorg, }}\right.$ and $\varepsilon$ ) were obtained by means of DFT calculations combined with the TB fitting procedure (see Section Electronic band structures). To correctly account for the impact of the cross electronic couplings on the hole mobilities, we devised a procedure to transform the model parameters in the Hamiltonian. More details on the mobility 
calculations and the transformation procedure can be found in the Supplementary Information.

\section{Code availability}

The codes used for calculating the electronic properties, i.e., Gaussion 09 (revision C.01), CRYSTAL14, and ADF2016, are commercially available. ${ }^{35,} 38,39$ The code used for the mobility calculations is available upon request to the authors.

\section{ACKNOWLEDGEMENTS}

We are grateful to Professor Osman Bakr and Dr. Dong Shi for providing the crystallographic data of the spiro-OMeTAD single crystal and for stimulating discussions. We thank Dr. Sean Ryno, Dr. Yuexing Zhang, and Stephen Shiring for helpful discussions and technical assistance. This work was supported by King Abdullah University of Science and Technology and ONR Global through Grant N62909-15-1-2003. Y.L. thanks partial support from the National Natural Science Foundation of China (Grant No. 21473102).

\section{AUTHOR CONTRIBUTIONS}

Y.L. conceived the idea. J.-L.B. directed the research. Y.L., H.Y.L., and C.Z. performed the calculations. Y.L., G.S., and J.-L.B. wrote the manuscript. All authors discussed and commented on the manuscript.

\section{ADDITIONAL INFORMATION}

Supplementary Information accompanies the paper on the npj Flexible Electronics website (doi:10.1038/s41528-017-0002-0).

Competing interests: The authors declare that they have no competing financial interests.

Publisher's note: Springer Nature remains neutral with regard to jurisdictional claims in published maps and institutional affiliations.

\section{REFERENCES}

1. Bach, U. et al. Solid-state dye-sensitized mesoporous $\mathrm{TiO}_{2}$ solar cells with high photon-to-electron conversion efficiencies. Nature 395, 583-585 (1998).

2. Kim, H. S. et al. Lead iodide perovskite sensitized all-solid-state submicron thin film mesoscopic solar cell with efficiency exceeding 9\%. Sci. Rep 2, 591 (2012).

3. Lee, M. M., Teuscher, J., Miyasaka, T., Murakami, T. N. \& Snaith, H. J. Efficient hybrid solar cells based on meso-superstructured organometal halide perovskites. Science 338, 643-647 (2012).

4. Green, M. A., Ho-Baillie, A. \& Snaith, H. J. The emergence of perovskite solar cells. Nat. Photon 8, 506-514 (2014).

5. Zhou, H. P. et al. Interface engineering of highly efficient perovskite solar cells. Science 345, 542-546 (2014).

6. Liu, M. Z., Johnston, M. B. \& Snaith, H. J. Efficient planar heterojunction perovskite solar cells by vapour deposition. Nature 501, 395-398 (2013).

7. Burschka, J. et al. Sequential deposition as a route to high-performance perovskite-sensitized solar cells. Nature 499, 316-319 (2013).

8. Cai, N. et al. An organic D- $\pi-A$ for record efficiency solid-state sensitized heterojunction solar cells. Nano. Lett. 11, 1452-1456 (2011).

9. Ameen, S. et al. Perovskite solar cells: influence of hole transporting materials on power conversion efficiency. ChemSusChem 9, 10-27 (2016).

10. Nguyen, W. H., Bailie, C. D., Unger, E. L. \& McGehee, M. D. Enhancing the holeconductivity of spiro-OMeTAD without oxygen or lithium salts by using spiro $(\mathrm{TFSI})_{2}$ in perovskite and dye-sensitized solar cells. J. Am. Chem. Soc. 136, 10996-11001 (2014).

11. Jeon, N. J. et al. o-Methoxy substituents in spiro-OMeTAD for efficient inorganicorganic hybrid perovskite solar cells. J. Am. Chem. Soc. 136, 7837-7840 (2014).

12. Leijtens, T., Lim, J., Teuscher, J., Park, T. \& Snaith, H. J. Charge density dependent mobility of organic hole-transporters and mesoporous $\mathrm{TiO}_{2}$ determined by transient mobility spectroscopy: implications to dye-sensitized and organic solar cells. Adv. Mater. 25, 3227-3233 (2013).

13. Xu, B. et al. A low-cost spiro[fluorene-9,9'-xanthene]-based hole transport material for highly efficient solid-state dye-sensitized solar cells and perovskite solar cells. Energy Environ. Sci. 9, 873-877 (2016).

14. Franckevicius, M. et al. A dopant-free spirobi[cyclopenta[2,1-b:3,4-b']dithiophene] based hole-transport material for efficient perovskite solar cells. Mater. Horiz. 2, 613-618 (2015).
15. Murray, A. T. et al. Modular design of spiro-OMeTAD analogues as hole transport materials in solar cells. Chem. Commun. 51, 8935-8938 (2015).

16. Ganesan, P. et al. A simple spiro-type hole transporting material for efficient perovskite solar cells. Energy Environ. Sci. 8, 1986-1991 (2015).

17. Shi, D. et al. Spiro-OMeTAD single crystals: remarkably enhanced charge-carrier transport via mesoscale ordering. Sci. Adv. 2, e1501491 (2016).

18. Podzorov, V. et al. Intrinsic charge transport on the surface of organic semiconductors. Phys. Rev. Lett. 93, 086602-1-086602-4 (2004).

19. Jurchescu, O. D., Baas, J. \& Palstra, T. T. M. Effect of impurities on the mobility of single crystal pentacene. Appl. Phys. Lett. 84, 3061-3063 (2004).

20. Yavuz, I. \& Houk, K. N. Mesoscale ordering and charge-transport of crystalline spiro-OMeTAD organic semiconductors. J. Phys. Chem. C 121, $993-999$ (2017).

21. Chi, W. J., Li, Q. S. \& Li, Z. S. Exploring the electrochemical properties of hole transport materials with spiro-cores for efficient perovskite solar cells from firstprinciples. Nanoscale 8, 6146-6154 (2016).

22. Alberga, D. et al. Theoretical investigation of hole transporter materials for energy devices. J. Phys. Chem. C 119, 23890-23898 (2015).

23. Kirkpatrick, J. \& Nelson, J. Theoretical study of the transfer integral and density of states in spiro-linked triphenylamine derivatives. J. Chem. Phys. 123, 0847031-084703-8 (2005).

24. Körzdörfer, T. \& Brédas, J. L. Organic electronic materials: recent advances in the DFT description of the ground and excited states using tuned range-separated hybrid functionals. Acc. Chem. Res. 47, 3284-3291 (2014).

25. Coropceanu, V. et al. Charge transport in organic semiconductors. Chem. Rev. 107, 926-952 (2007).

26. Slater, J. C. \& Koster, G. F. Simplified LCAO method for the periodic potential problem. Phys. Rev 94, 1498-1524 (1954).

27. Ryno, S. M., Risko, C. \& Brédas, J. L. Impact of molecular packing on electronic polarization in organic crystals: the case of pentacene vs TIPS-pentacene. J. Am. Chem. Soc. 136, 6421-6427 (2014).

28. Stone, A. The Theory of Intermolecular Forces (Oxford University Press, 2013).

29. Shuai, Z., Geng, H., Xu, W., Liao, Y. \& André, J. M. From charge transport parameters to charge mobility in organic semiconductors through multiscale simulation. Chem. Soc. Rev. 43, 2662-2679 (2014).

30. Li, H. Y., Qiu, Y. \& Duan, L. Multi-scale calculation of the electric properties of organicbased devices from the molecular structure. Org. Electron 33, 164-171 (2016).

31. Seki, K. \& Tachiya, M. Electric field dependence of charge mobility in energetically disordered materials: polaron aspects. Phys. Rev. B 65, 014305-1-014305-13 (2001).

32. Marcus, R. A. Electron transfer reactions in chemistry. Theory and experiment. Rev. Mod. Phys. 65, 599-610 (1993).

33. da Silva Filho, D. A., Kim, E. G. \& Brédas, J. L. Transport properties in the rubrene crystal: electronic coupling and vibrational reorganization energy. Adv. Mater. 17, 1072-1076 (2005)

34. Coropceanu, V. et al. Hole- and electron-vibrational couplings in oligoacene crystals: intramolecular contributions. Phys. Rev. Lett. 89, 275503-1-275503-4 (2002).

35. Frisch, M. J. et al. Gaussion 09, revision C.01 (Gaussian Inc., 2011)

36. Chai, J. D. \& Head-Gordon, M. Long-range corrected hybrid density functionals with damped atom-atom dispersion corrections. Phys. Chem. Chem. Phys. 10, 6615-6620 (2008).

37. Sun, H. et al. Ionization energies, electron affinities, and polarization energies of organic molecular crystals: quantitative estimations from a polarizable continuum model (PCM)-tuned range-separated density functional approach. J. Chem. Theory. Comput. 12, 2906-2916 (2016).

38. Dovesi, R. et al. CRYSTAL14 User's Manual (University of Torino, 2014).

39. ADF (2016), SCM http://www.scm.com.

40. te Velde, G. et al. Chemistry with ADF. J. Comput. Chem. 22, 931-967 (2001).

41. Fonseca Guerra, C., Snijders, J. G., te Velde, G. \& Baerends, E. J. Towards an orderN DFT method. Theor. Chem. Acc. 99, 391-403 (1998).

Open Access This article is licensed under a Creative Commons Attribution 4.0 International License, which permits use, sharing, adaptation, distribution and reproduction in any medium or format, as long as you give appropriate credit to the original author(s) and the source, provide a link to the Creative Commons license, and indicate if changes were made. The images or other third party material in this article are included in the article's Creative Commons license, unless indicated otherwise in a credit line to the material. If material is not included in the article's Creative Commons license and your intended use is not permitted by statutory regulation or exceeds the permitted use, you will need to obtain permission directly from the copyright holder. To view a copy of this license, visit http://creativecommons. org/licenses/by/4.0/.

(c) The Author(s) 2017 